CAHIERS DE

NARRATOLOGIE

\section{Cahiers de Narratologie}

Analyse et théorie narratives

18 | 2010

Littérature et sciences

\title{
Des images et des paraboles : Niels Bohr et le discours descriptif en physique quantique
}

\author{
Ilias Yocaris
}

\section{(2)enEdition}

Journals

Édition électronique

URL : https://journals.openedition.org/narratologie/6025

DOI : 10.4000/narratologie.6025

ISSN : 1765-307X

Éditeur

LIRCES

\section{Référence électronique}

Ilias Yocaris, «Des images et des paraboles : Niels Bohr et le discours descriptif en physique quantique », Cahiers de Narratologie [En ligne], 18 | 2010, mis en ligne le 05 janvier 2011, consulté le 21 septembre 2021. URL : http://journals.openedition.org/narratologie/6025 ; DOI : https://doi.org/ $10.4000 /$ narratologie.6025

Ce document a été généré automatiquement le 21 septembre 2021.

\section{cc) (†) $\ominus$}

Cahiers de Narratologie - Analyse et théorie narratives est mis à disposition selon les termes de la licence Creative Commons Attribution - Pas d'Utilisation Commerciale - Pas de Modification 4.0 International. 


\title{
Des images et des paraboles : Niels Bohr et le discours descriptif en physique
}

\section{quantique}

\author{
Ilias Yocaris
}

[I]l faut que les éléments de la multiplicité n'aient ni forme sensible ni signification conceptuelle, ni dès lors fonction assignable. Ils n'ont même pas d'existence actuelle, et sont inséparables d'un potentiel ou d'une virtualité. C'est en ce sens qu'ils n'impliquent aucune identité préalable, aucune position d'un quelque chose qu'on pourrait dire un ou le même; mais au contraire leur indétermination rend possible la manifestation de la différence en tant que libérée de toute subordination.

1 Cette étude porte sur l'importance accordée aux images verbales dans le discours descriptif utilisé en mécanique quantique, et plus précisément sur la conception de la langue scientifique qui est celle de Niels Bohr (1885-1962): en raison d'une série de considérations techniques, méthodologiques et épistémologiques que nous nous proposons d'analyser in extenso, Bohr considère effectivement que les phénomènes subatomiques ne peuvent être évoqués directement (sans référence au contexte observationnel), par le biais d'un langage dénotatif non figural, mais uniquement de manière métaphorique, détournée, ce qui réduit à ses yeux le discours descriptif des physiciens à « des images et des paraboles ». En examinant les textes de Bohr à la lumière d'un certain nombre de travaux épistémologiques, de commentaires et d'expérimentations auxquels ils ont donné lieu ultérieurement, nous nous proposons de 
décrire les implications conceptuelles d'une telle prise de position, qui constitue une vraie révolution sur le plan philosophique.

\section{Des « objets » qui échappent aux « formes pures de l'intuition sensible »}

2 Quel est le problème fondamental auquel se trouvent confrontés les fondateurs de la mécanique quantique, à savoir Bohr, Heisenberg, Born, Pauli et Schrödinger? En termes kantiens, la physique quantique met au jour un niveau de réalité qui échappe aux «formes pures de l'intuition sensible» (Kant $1980:$ 783-784), l'espace et le temps ${ }^{1}$. De ce fait, les micro-objets quantiques, contrairement aux objets macrophysiques peuplant le monde tel que nous le percevons à notre échelle, ne peuvent être pensés comme des substances permanentes ayant une existence et des caractéristiques intrinsèques: les microparticules ont un comportement phénoménal discontinu, et la plupart des propriétés qu'on leur attribue ne sont (sauf dans certains cas précis) valables que par rapport à un contexte expérimental donné. On dira donc (avec le physicien Bernard d'Espagnat) que l'«objet» quantique n'a pas une «objectivité forte» mais une «objectivité faible ${ }^{2}$ ». D'où l'insistance de Bohr et de ses épigones sur l'idée que la mécanique quantique est fondée dans sa totalité sur une rupture historique avec le dualisme cartésien sujet/objet, au profit d'une vision intersubjectiviste et holistique du « réel» microphysique (v. notamment Bohr $1991: 207,257-258$, d'Espagnat $1985: 31,60$, n. 1, 298 n. 1, Zeilinger 1997). En quoi consiste une telle vision? Du fait qu'il ne saurait être considéré comme une « substance " permanente, l'« objet » quantique ne préexiste pas à proprement parler à son observation! La grande nouveauté de la mécanique quantique, c'est de donner à voir des « objets » radicalement « contextuels » (v. Bitbol 1998: 311 et passim) qui ne correspondent pas vraiment à des «étants» ayant une existence intrinsèque, mais plutôt à « des déterminations et des événements « relatifs » à diverses classes de dispositifs expérimentaux» (Bitbol 1996: 28; italiques de l'auteur, comme dans toutes les citations qui suivent) ${ }^{3}$. Ainsi par exemple des microparticules comme les photons peuvent présenter dans certains types d'expériences un comportement corpusculaire, mais dans d'autres un comportement ondulatoire (v. p. ex. Lochak et al. 1989 : 21-43).

3 Les fondateurs de la mécanique quantique se heurtent donc à une série de difficultés techniques et ontologiques à première vue insurmontables, puisque le formalisme qu'ils ont eux-mêmes élaboré "ne permet pas de description intuitive [des "objets» microphysiques] sous les formes habituelles » (Bohr 1991 : 256). Or, ces difficultés ont un impact déterminant sur leur conception du «langage» descriptif utilisé en physique. Bohr ne cesse ainsi de souligner que le fonctionnement référentiel dénotatif et "décontextualisé » (Bitbol 1996: 46) de la langue "ordinaire», qui était la base de la description du monde proposée par la physique " classique » (de Newton à Einstein), est inadéquat pour rendre compte de l'« objet » quantique. La raison en est que ce type de fonctionnement présuppose a priori la possibilité d'une re-connaissance indéfiniment répétable des « entités » décrites, et ce dans n'importe quel contexte observationnel. C'est précisément cette possibilité qui disparaît (v. Bitbol 1996 : 26-54) à cause de l'« objectivité faible » du « référent » en mécanique quantique : ce dernier n'est plus réidentifiable par continuité spatio-temporelle et de ce fait on ne saurait utiliser pour le décrire un « langage objectiviste» (d'Espagnat 2002: 26) dont «la forme grammaticale permet au 
moins de penser ce dont il traite [...] comme existant indépendamment de nous-mêmes " (ibid.). Ce qui entrave en somme l'utilisation de la langue "ordinaire", c'est que nous n'avons plus affaire à un objet isolable qui préexisterait à son observation et posséderait des caractéristiques intrinsèques mais à une suite d'événements dont chacun est constitutivement déterminé par les circonstances de son émergence (le dispositif observationnel utilisé pour effectuer une expérience donnée). Or, le fonctionnement référentiel et syntaxique de la langue « ordinaire » impose par définition à notre esprit l'image d'un objet fixe, clairement délimité et porteur de propriétés intrinsèques, un objet qui serait le substrat invariable de déterminations, elles, variables (v. Bitbol 2000a : 78) : ainsi on tend à se figurer spontanément le référent du mot « électron » comme un petit corpuscule, ressemblant aux grains de poussière que nous pouvons voir dans la lumière. L'«objet" quantique ne correspondant absolument pas à une telle schématisation verbale, il faut dès lors trouver des stratégies référentielles «alternatives » pour le décrire. Le cas des photons constitue à cet égard l'exemple le plus frappant, et nous servira donc de fil directeur pour notre démonstration.

4 Les photons peuvent être définis comme des «entité[s] énergétique[s] non dissociables qui intervien[nen]t dans tout échange d'énergie entre la lumière et la matière » (Lochak et al. 1989 : 219). Or, du fait de la dimension «contextuelle» de ces «entités», leur comportement change du tout au tout en fonction du dispositif expérimental utilisé pour étudier leurs " propriétés » : dans certains types d'expériences, les photons adoptent ainsi l'équivalent approximatif d'un comportement corpusculaire (ils se présentent plus ou moins comme des « objets discrets »), et on peut alors a priori essayer de les localiser dans une région délimitée de l'espace; dans d'autres, ils adoptent l'équivalent approximatif d'un comportement ondulatoire (ils semblent «se propager » dans l'espace à la manière des ondes d'un liquide), et leur description doit alors tenir compte de certains phénomènes (diffraction, interférences, mise en phase...) qui les rendent "non localisables » et indiscernables les uns des autres. On se trouve dès lors face à une «dialectique de propriétés localisées et de propriétés délocalisées» (Lochak et al. 1989: 204) qui a suscité la perplexité des fondateurs de la mécanique quantique, et notamment de Bohr. Dans un article de 1949, ce dernier constate ainsi que les « objets atomiques » en général (et les photons en tout premier lieu) ne peuvent être décrits de manière non ambiguë par "des mots [comme "onde» ou «particule»], adaptés aux images de la physique classique» (Bohr 1991: 209), et ce parce que «les résultats obtenus dans des conditions expérimentales différentes ne peuvent être englobés en une seule image " (Bohr 1991 : 208) : en clair, du fait de leur « contextualité », les micro-objets quantiques ne constituent pas des « "groupes" stables de perceptions (ou impressions)» (d'Espagnat 2002 : 409) susceptibles de faire l'objet d'une représentation unitaire. Cependant, Bohr reste persuadé en même temps que les physiciens ne peuvent en aucune manière renoncer au langage descriptif hérité de la physique classique pour rendre compte des phénomènes auxquels ils sont confrontés. Pourquoi ? Du fait justement qu'il s'appuie sur notre expérience « intuitive » du monde qui nous entoure (le « réel » macrophysique), ce langage est pour lui le seul apte sur le plan communicationnel à transmettre nos connaissances sur les « objets quantiques » :

d'aussi loin que les phénomènes puissent transcender le domaine des explications de la physique classique, la description de tous les résultats de l'expérience doit être exprimée en termes classiques. La raison en est simple: par le mot d'«expérience", nous nous référons à une situation où nous pouvons dire à d'autres hommes ce que nous avons fait et ce que nous avons appris; il en résulte 
que la description du dispositif expérimental et des résultats des observations doit être exprimée en un langage dénué d'ambiguïté, se servant convenablement de la terminologie de la physique classique » (Bohr 1991 : 207 ; cf. 257).

\section{Un discours descriptif purement figural}

Comment résoudre ce problème a priori insoluble ? Pour rendre compte des particularités $\mathrm{du}$ « réel » microphysique sans renoncer à «la terminologie de la physique classique ", Bohr choisit précisément d'utiliser un discours descriptif purement figural. En quoi consiste le fonctionnement de ce discours ? Dès 1927, Bohr introduit le fameux concept de " complémentarité », qui s'avèrera extrêmement fécond sur le plan philosophique même si sa pertinence d'un strict point de vue scientifique nous parait aujourd'hui douteuse (v. Lévy-Leblond $2000: 254)$ : des «objets » comme les photons ne peuvent être décrits que par une juxtaposition de traits définitionnels « complémentaires ", à la fois incompatibles et indissociables, ce qui revient concrètement à leur attribuer (à la suite de Louis de Broglie : v. p. ex. de Broglie $1963: 69,113,1937: 51$ ) une nature à la fois corpusculaire et ondulatoire. Comme le souligne le grand physicien John S. Bell, l'« objet» quantique apparaît ainsi comme une entité dont aucune représentation unitaire n'est possible, dans la mesure où on lui attribue in fine des caractéristiques contradictoires :

There is very little I can say about "complementarity". But I wish to say one thing. It seems to me that Bohr used this word with the reverse of its usual meaning. Consider for example the elephant. From the front she is head, trunk and two legs. From the back she is bottom, tail, and two legs. From the sides she is otherwise, and from top and bottom different again. These various views are complementary in the usual sense of the word. They supplement one another, they are consistent with one another, and they are all entailed by the unifying concept elephant. It is my impression that to suppose Bohr used the word "complementary" in this ordinary way would have been regarded by him as missing his point and trivializing his thought. He seems to insist rather that we must use in our analysis elements which contradict one another, which do not add up to, or derive from, a whole. By "complementarity" he meant, it seems to me, the reverse : contradictoriness. (Bell 1989 : 363-364). 
Fig. 1

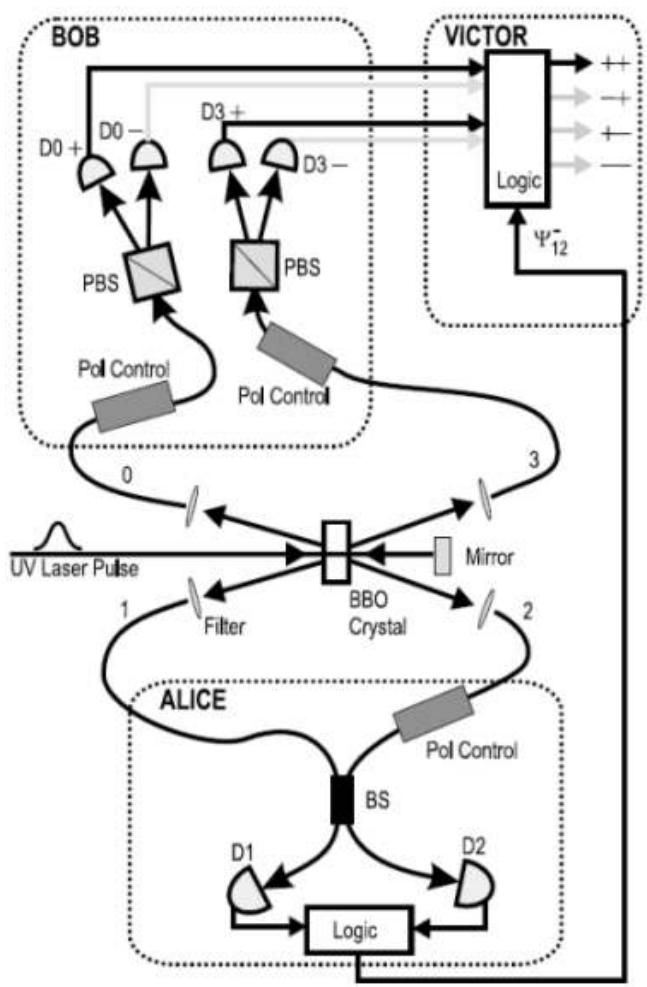

Dispositif expérimental utilisé pour observer le comportement de deux paires de photons « intriqués » (source : Jennewein et al. 2002)

C'est cette juxtaposition de représentations mutuellement incompatibles pour décrire de manière détournée un « objet » en soi problématique qui confère justement au discours descriptif utilisé par bon nombre de fondateurs de la mécanique quantique sa dimension figurale. Pourquoi ? En termes linguistiques, Bohr attribue en définitive aux photons des prédicats contradictoires (v. p. ex. Chevalley 1991: 88 et passim), puisque sa démarche revient en quelque sorte à émettre un jugement de ce type : « $x$ (un photon donné) est à la fois $y$ (une "onde") et $\sim y$ (une "particule") ». Dès lors, le « hiatus » référentiel et logique ainsi occasionné (le récepteur du message n'arrivant absolument pas à se figurer un tel « objet ») reproduit indirectement, par une sorte d' « homomorphisme ${ }^{4}$ ", la discontinuité qui caractérise le comportement des photons " eux-mêmes ", en raison de leur dimension « contextuelle»! En somme, donc, les photons ne sont ni des ondes ni des particules (v. Balibar et Lévy-Leblond 1984 : 66-68), mais la juxtaposition d'une image corpusculaire et d'une image ondulatoire pour les décrire suggère métaphoriquement qu'il s'agit là d'« entités » qui échappent entièrement à notre mode de représentation intuitif : comme le souligne Catherine Chevalley,

[1]es prédicats contradictoires sont en réalité attribués à plusieurs manifestations phénoménales d'un objet qui, n'étant pas lui-même représentable, ne souffre pas d'être le lieu d'une « coïncidence des opposés » (Chevalley $1991: 88$ ).

Dès lors, le discours des physiciens se réduit fatalement à " des images et des paraboles » (N. Bohr, cité in Heisenberg 1972 : 285), d'autant que les phénomènes appelant (au-delà $\mathrm{du}$ principe de complémentarité stricto sensu) des descriptions intrinsèquement oxymoriques de ce genre sont légion ${ }^{5}$. 


\section{Un bouleversement épistémologique sans précédent}

la physique sont très importantes : le recours délibéré à une stratégie descriptive axée sur la juxtaposition métaphorique de représentations mutuellement incompatibles entraîne un vrai bouleversement sur le plan épistémologique, et ce pour plusieurs raisons.

(1) Il plonge la physique dans une véritable «crise de mots " (Chevalley 1991 : 92), dont elle n'est toujours pas sortie (v. p. ex. Lochak et al. 1989: 61, Lévy-Leblond 2000). En insistant sur la «contextualité" des phénomènes microphysiques, la dimension métaphorique des «mots adaptés aux images de la physique classique » et la nécessité d'attribuer à l'« objet quantique » des prédicats contradictoires, Bohr touche un point très sensible, dans la mesure où il remet de facto en question deux postulats de la philosophie rationaliste qui semblaient inébranlables: (a) l'intelligibilité du réel en fonction des catégories kantiennes de la connaissance (causalité, subsistance, unité/ pluralité etc ${ }^{6}$.) ; (b) l'obligation de décrire les «objets » en fonction d'une logique non contradictoire, du type aristotélicien.

(2) Il donne à voir une conception non plus « analytique », mais « synthétique » de l'objet de référence (v. Chevalley 1991: 97). En effet, contrairement à bon nombre de ses prédécesseurs, Bohr ne considère pas le langage comme un « reflet » ou une «copie » du réel, mais comme un instrument de formalisation active de celui-ci (v. Chevalley 1991 : 94 et passim). Du fait qu'ils drainent avec eux tout un ensemble de représentations relevant de notre perception intuitive du monde qui nous entoure, les mots utilisés pour décrire les phénomènes quantiques ne sont pas neutres et transparents sur le plan ontologique, mais véhiculent toujours une perspective observationnelle particulière projetée sur les «objets» considérés (Chevalley 1991: 9477). Dès lors, pour décrire ces «objets» de manière adéquate, il faut juxtaposer métaphoriquement plusieurs points de vue descriptifs différents (v. Bitbol 2000a: 266), créant ainsi «une sorte de vision stéréoscopique ( Ricoeur 1975: 290) qui découle d'un "assemblage» de données perceptuelles et/ou expérimentales a priori irréductibles à toute unification.

(3) Il implique le renoncement à un idéal régulateur vieux comme la science, à savoir la quête d'une représentation à la fois totalisante et « objective » du réel à partir d'un point de vue observationnel «surplombant »: tout comme le principe d'indétermination, la complémentarité bohrienne montre que la réalité possède une richesse et une complexité telles qu'elles débordent «chaque langage, chaque structure logique, chaque éclairage conceptuel ; chacun peut seulement en exprimer - mais réussit à en exprimer - une partie » (Prigogine et Stengers $1986: 313$ ).

\section{Une multitude de stratégies référentielles}

Quelles sont les conclusions que l'on peut tirer de la fabuleuse expérience bohrienne? Confrontés aux difficultés colossales soulevées par l'interprétation du formalisme quantique, les épigones de Bohr (toutes tendances confondues) ont le choix entre plusieurs démarches méthodologiques - et, par extension, plusieurs stratégies référentielles - différentes : 
13 (1) Maintenir plus ou moins en l'état le cadre conceptuel esquissé par Bohr, en considérant comme acquis le fait que le langage "ordinaire» ne peut représenter directement les microparticules, mais doit néanmoins être utilisé pour les décrire. D’un point de vue strictement linguistique (et stylistique !), un tel choix peut impliquer :

Un dédoublement énonciatif constant du discours descriptif. On recourt dans ce cas à une série de précisions méta-énonciatives soulignant la dimension figurale du vocabulaire utilisé : ainsi par exemple un néo-bohrien comme Bernard d'Espagnat évoque explicitement l'« armature métaphorique » (d'Espagnat $2002: 431$ ) de son propre discours descriptif, ou encore la dimension "semi-allégorique» (d'Espagnat 2002: 59) de la terminologie utilisée par les adeptes du formalisme feynmanien.

Une description a contrario des « objets » et des phénomènes microphysiques, partant du principe que les prédictions observationnelles fournies par le formalisme quantique ne nous révèlent pas ce qu'ils sont, mais tout au plus ce qu'ils ne sont pas (d'où l'élaboration de concepts clés comme l'objectivité "faible", la "non"-séparabilité ou la "non"-localité : v. d'Espagnat 1994 : 141-144, d'Espagnat 2002 : 93, 434, 440, 447).

L'utilisation de néologismes permettant d'étendre le domaine d'application du langage dénotatif, en y incluant des «entités» non représentables intuitivement et/ou susceptibles de se voir attribuer le prédicat... "impossibilité de toute prédication univoque ». Jean-Marc Lévy-Leblond (2000: 260-263) propose ainsi de remplacer les hyponymes «bosons » et « fermions » (désignant les deux grandes familles de particules quantiques) par leur hyperonyme "quantons », le concept de "non-séparabilité » par

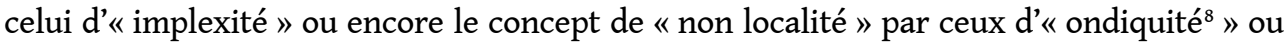
de « pantopie ${ }^{9}$ ».

Bien entendu, ces options ne sont pas forcément exclusives les unes des autres.

(2) Revenir délibérément au système de représentations « locales » hérité de la physique classique. Le moins qu'on puisse dire, c'est que, en raison des évidences expérimentales, cette option se trouve à l'heure actuelle plus ou moins marginalisée. Seuls quelques physiciens isolés comme Franco Selleri (1994: 174-184) défendent encore une approche de ce type, mais leurs motivations semblent à vrai dire plus idéologiques que cognitives.

19 (3) Renoncer (temporairement ou définitivement) au langage verbal pour décrire l'univers microphysique, en s'en tenant strictement au formalisme logico-mathématique mis au point par les fondateurs de la mécanique quantique et leurs épigones (Dirac, Birkhoff, von Neumann ...) ou bien en misant sur des innovations théoriques susceptibles à terme d'amener un changement de paradigme en physique [v. à titre indicatif Hélein et Kouneiher 2002, Baird et al. (dirs), 2008]. De manière prévisible, c'est la voie choisie par certains mathématiciens comme Roger Penrose, qui proposent une conception platonisante $\mathrm{du}$ « réel » microphysique ${ }^{10}$ tout à fait aux antipodes de la pensée bohrienne (v. Chevalley $1991: 104)$.

20 (4) Élaborer des logiques «trivalentes» (les fameuses «logiques quantiques») permettant de supprimer le principe du tertium non datur, ce qui rend possible d'attribuer littéralement au même "objet » des prédicats contradictoires (v. à titre indicatif Reichenbach 1944, Destouches-Février 1951, Putnam 1968).

21 (5) Évacuer purement et simplement le problème de la référence, en continuant d'utiliser (au prix d'approximations plus ou moins licites selon les phénomènes considérés : v. Bitbol 1998 : 207) le vocabulaire descriptif hérité de la physique classique. On n'hésitera pas ainsi à parler dans les manuels de référence (v. p. ex. Cohen-Tannoudji, Laloë et Liu 
1973) de "particules», de "propriétés", de «trajectoires» etc., alors que le profil sémique de ces mots se trouve en réalité profondément altéré : dans le domaine disciplinaire de la mécanique quantique, un vocable comme "particule» réalise (ou plutôt peut réaliser, en fonction du positionnement épistémologique du physicien qui l'utilise, du contexte expérimental de référence, du sous-domaine considéré ${ }^{11}$ etc.) des sèmes comme /indiscernabilité/, /enchevêtrement/ (le fameux quantum entanglement) ou /ubiquité/, alors que, inversement, il peut cesser de véhiculer le sème /aspect corpusculaire/... Bien entendu, passer outre ces difficultés sémantiques ${ }^{12}$ et adopter (comme le fait à l'heure actuelle l'immense majorité des physiciens qui travaillent « sur le terrain ») un «langage multitudiniste» (d'Espagnat 2002: 24) devenu en réalité entièrement conventionnel équivaut in fine à faire de la mécanique quantique « un jeu de recettes de calcul, dévoré par l'absence de l'objet » (Chevalley 1991: 89).

(6) Radicaliser à l'extrême la vision « contextuelle » du « réel » microphysique qui était déjà - en partie - celle de Bohr, en postulant que le langage utilisé pour décrire les particules (sub)atomiques ne renvoie pas (fût-ce métaphoriquement) à ces particules " elles-mêmes ", mais à un ensemble de normes fonctionnelles, d'invariants processuels d'ordre gestuel, méthodologique et/ou symbolique et de présuppositions conceptuelles qui déterminent constitutivement l'activité des physiciens, les régularités expérimentalement observables découlant in fine d'un "processus de co-définition des activités et des formes sur lesquelles elles s'exercent» (Bitbol 1998: 151). Cette option, qui revient à adopter en définitive une conception autoréflexive du discours descriptif dans les sciences en général, est défendue non sans brio par des épistémologues comme Hervé Zwirn (2000) ou Michel Bitbol (1996, 1998, 2000b). Le moins qu'on puisse dire, toutefois, c'est qu'elle ne suscite pas l'enthousiasme des physiciens, pour des raisons que l'on comprendra assez aisément...

\section{Conclusion}

23 L'avènement de la mécanique quantique révèle sans doute pour la première fois que le langage descriptif de la physique ne constitue pas un simple « reflet » du monde qui nous entoure (et dont nous faisons partie intégrante), mais informe activement notre vision du « réel » : l'insistance de Bohr sur le fait que ce langage est littéralement imprégné par une série "d'images et de représentations qui se réfèrent aux événements de la vie quotidienne» (Bohr 1991: 252) montre assez qu'à ses yeux il a une dimension proprement modélisante, qui va bien au-delà de son fonctionnement strictement dénotatif. Or, les paradoxes irréductibles auxquels on se heurte dès qu'on essaie de décrire l'univers quantique en fonction du système de représentations hérité de la physique " classique » laissent entrapercevoir les limites d'une telle démarche sur le plan ontologique: rien (autrement dit, aucune instance métaphysique semblable au Dieu cartésien qui ne saurait vouloir nous tromper) ne garantit en fait la pertinence de notre activité modélisante, puisqu'on ne saurait affirmer avec certitude à l'heure actuelle (a) que «les mots dont nous disposons - entendons les concepts que ceux-ci désignent correspondent de façon bi-univoque à des "moellons" de la réalité » (d'Espagnat 2002 : 227) et (b) que les structures mêmes du langage verbal et/ou logico-mathématique utilisé par les physiciens correspondent par isomorphisme (ou, à tout le moins, par homomorphisme ${ }^{13}$ ) aux linéaments d'un aliquid préstructuré indépendamment de nos aptitudes perceptionnelles et des normes qui régissent nos activités expérimentales (v. 
d'Espagnat 2002 : 167, 427-428, 452, 499 n. 1, 518). La description raisonnée du " réel » microphysique (quel que soit le sens de cette expression) est donc pour nous un perpétuel défi, dans la mesure où elle nécessite de notre part un effort d'adaptation constant sur le plan conceptuel et discursif afin de verbaliser l'indicible et de penser l'impensable.

\section{BIBLIOGRAPHIE}

Allén S. (éd.) (1989). Possible worlds in humanities, arts and sciences. Proceedings of Nobel Symposium 65, Berlin/New York : de Gruyter.

Aspect A. et al. (1982a). « Experimental realization of Einstein-Podolsky-Rosen

gedankenexperiment ; a new violation of Bell's inequalities », Physical Review Letters, 49, pp. 91-94.

Aspect A. et al. (1982b). « Experimental test of Bell's inequalities using time-varying analyzers », Physical Review Letters, 49, pp. 1804-1807.

Baird P. et al. éds(2008). Systèmes intégrables et théorie des champs quantiques, Paris : Hermann.

Bakhtine M. (1978). Esthétique et théorie du roman, trad. du russe par Daria Olivier, Paris : Gallimard. Balibar F. et Lévy-Leblond J.-M. (1984). Quantique : rudiments, Paris : InterÉditions.

Bell J. S. (1989). « Six possible worlds for quantum mechanics », in Allén S. éd. 1989 : 359-373.

Bitbol M. et Laugier S. éds (1997). Physique et réalité. Un débat avec Bernard d'Espagnat, Paris : Frontières-Diderot.

Bitbol M. (1996). Mécanique quantique. Une introduction philosophique, Paris : Flammarion.

-(1998). L'Aveuglante proximité du réel, Paris : Flammarion.

-(2000a). Physique et philosophie de l'esprit, Paris : Flammarion.

-(2000b). «Physique quantique et cognition », Revue internationale de philosophie, 54, 212, La mécanique quantique, pp. 299-328.

Bohm D. (1987). La Plénitude de l'univers, trad. de l'anglais par Tchalaï Unger, Monaco : Le Rocher. Bohm D. et Hiley B. J. (1993). The Undivided universe, Londres : Routledge \& Kegan Paul.

Bohr N. (1935). « Can quantum mechanical description of physical reality be considered complete?», Physical Review, 48, pp. 696-702.

-(1991). Physique atomique et connaissance humaine, trad. de l'anglais par Edmond Bauer et Roland Omnès, Catherine Chevalley éd., Paris. Gallimard.

de Broglie L. (1937). Matière et lumière, Paris : Albin Michel.

-(1963 [11924]). Recherches sur la théorie des quanta, Paris : Masson.

Chevalley C. (1991). « Le Dessin et la couleur », introduction de Bohr N., 1991, pp. 17-140.

-(1991'). « Glossaire », annexe de Bohr N., 1991, pp. 345-567.

-(1992). « Physique quantique et philosophie », Le Débat, 72, pp. 65-76. 
-(1995). « Nature et loi dans la philosophie moderne », in Notions de philosophie, Kambouchner D.

(éd.), Paris : Gallimard, pp. 127-230.

-(1996). « Physics as an art : the German tradition and the symbolic turn in philosophy, history of art and natural science in the 1920 s", in Tauber A. I. (éd.), The Elusive synthesis : aesthetics and science, Dordrecht/Boston/London : Kluwer, pp. 227-249.

-(1998). «Introduction », in Heisenberg W., 1998 : 17-245.

Cohen-Tannoudji C. Laloë F. et Liu B. (1973). Mécanique quantique, tome 1, Paris : Hermann.

Destouches-Février P. (1951). La Structure des théories physiques, Paris : PUF.

Einstein A., Podolsky B. et Rosen N. (1935). « Can quantum mechanical description of physical reality be considered complete?", Physical Review, 47, pp. 777-780.

Esfeld M. (2001). Holism in philosophy of mind and philosophy of physics, Dordrecht : Kluwer.

d'Espagnat B. (1985). Une Incertaine réalité. Le monde quantique, la connaissance et la durée, Paris : Gauthier-Villars.

-(1994). Le Réel Voilé. Analyse des concepts quantiques, Paris : Fayard.

-(2002). Traité de physique et de philosophie, Paris : Fayard.

d'Espagnat B. (dir.) (2002). Implications philosophiques de la science contemporaine, tome 2, Les Interprétations de la physique quantique, Paris : PUF.

Halder M. et al. (2005). « Photon-bunching measurement after two 25-km-long optical fibers », Physical review A, 71, 4, pp. 042335-1-042335-5, www.gapoptique.unige.ch/Publications/PDF/ Bunching25km.pdf (4 février 2009).

Heisenberg W. (1952). Philosophic problems of nuclear science, trad. de l'allemand par F. C. Hayes, Londres : Faber \& Faber.

-(1962). La Nature dans la physique contemporaine, trad. de l'allemand par Ugné Karvélis et A. E. Leroy, Paris : Gallimard.

-(1972). La Partie et le tout. Le monde de la physique atomique, trad. de l'allemand par Paul Kessler, Paris : Albin Michel.

-(1998). Philosophie. Le manuscrit de 1942, trad. de l'allemand par Catherine Chevalley, Paris : Seuil. Hélein F. et Kouneiher J. (2002). « Finite dimensional Hamiltonian formalism for gauge theory and quantum field theory ", Journal of Mathematical Physics, 43, 5, pp. 2306-2347.

Jammer M. (1974). The Philosophy of Quantum Mechanics, New York : John Wiley and Sons.

Jennewein T. et al. (2002). « Experimental nonlocality proof of quantum teleportation and entanglement swapping ", Physical Review Letters, 88, 1, pp. 017903-1-017903-4.

Kant I. (1980). Euvres philosophiques, trad. de l'allemand par Ferdinand Alquié et al., tome I, Paris : Gallimard, coll. «Bibliothèque de la Pléiade ».

(1985). Cuvres philosophiques, trad. de l'allemand par Ferdinand Alquié et al., tome II, Paris : Gallimard, coll. « Bibliothèque de la Pléiade ».

(1986). CEuvres philosophiques, trad. de l'allemand par Ferdinand Alquié et al., tome III, Paris : Gallimard, coll. « Bibliothèque de la Pléiade ». 
Lévy-Leblond J.-M. (2000). « Mots \& maux de la physique quantique. Critique épistémologique et problèmes terminologiques ", Revue internationale de philosophie, 54, 212, La mécanique quantique, pp. 243-265.

Lochak G. et al. (1989). L'Objet quantique. Comment l'esprit vient aux atomes, Paris : Flammarion.

Penrose R. (1992). L'Esprit, l'ordinateur et les lois de la physique, trad. de l'anglais par Françoise Balibar et Claudine Tiercelin, Paris : InterÉditions.

Prigogine I. et Stengers I. (1986 [11979]). La Nouvelle alliance. Métamorphose de la science, Paris : Gallimard.

Prigogine I. (1996). La Fin des certitudes, Paris : Odile Jacob.

Putnam H. (1968). « Is Logic Empirical ? », in Robert S. Cohen et Marx W. Wartofsky (éds), Boston Studies in the Philosophy of Science, vol. 5, Dordrecht : Reidel, pp. 216-241.

Reichenbach H. (1944). Philosophic foundations of quantum mechanics, Berkeley et Los Angeles : University of California Press.

Ricoeur P. (1975). La Métaphore vive, Paris : Seuil.

Schrödinger E. (1992). Physique quantique et représentation du monde, Paris : Seuil.

Selleri F. (1994). Le Grand débat de la théorie quantique, Paris : Flammarion.

Walliser B. (1977). Systèmes et modèles. Introduction critique à l'analyse de systèmes, Paris : Seuil.

Walther P. et al. (2005). « Experimental violation of a cluster state Bell inequality », Physical Review Letters, 95, pp. 020403-1-020403-4, arxiv.org/pdf/quant-ph/0507086v1 (4 février 2009).

Whitaker A. (1996). Einstein, Bohr and the quantum dilemma, Cambridge : Cambridge University Press.

Zeh H.-D. (1993). « There are no quantum jumps, nor are there particles! », Physics letters, 172, 4, pp. 189-192.

Zeilinger A. et al. (1997). « Experimental quantum teleportation », Nature, 390, pp. 575-579.

Zeilinger A. (1997). « On the interpretation and philosophical foundation of quantum mechanics ", http://www.quantum.univie.ac.at/zeilinger/philosop.html (8 octobre 2008).

Zeilinger A. et al. (2008a). « Bell theorem without inequalities for two particles. I. Efficient detectors », Physical Review A, 78, 022101-1-022110-7.

Zeilinger A. et al. (2008b). « Bell theorem without inequalities for two particles. II. Inefficient detectors ", Physical Review A, 78, 022111-1-022111-12.

Zwirn H. (2000). Les Limites de la connaissance, Paris : Odile Jacob.

\section{NOTES}

1. Voir par exemple dans notre bibliographie Bohr 1991 : 255 et passim, Chevalley 1995 etc. Bien entendu, nous suivons ici l'interprétation «orthodoxe» de la mécanique quantique (interprétation dite aussi « de Copenhague », du nom de la ville où elle fut mise au point par Niels Bohr à la fin des années 1920). Cette interprétation a certes (il ne faut pas l'oublier) donné lieu à une interminable série de controverses épistémologiques, qui culminèrent dans les années 1990 [v. Jammer 1974, d'Espagnat 1985, Chevalley 1991, 1991', Penrose 1992, d'Espagnat 1994, Selleri 1994, Bitbol 1996, Prigogine 1996, Whitaker 1996, Bitbol et Laugier (éds) 1997, Bitbol 1998, 
Chevalley 1998, Esfeld 2001, d'Espagnat 2002, d'Espagnat (dir.) 2002 etc.]. Il n'en reste pas moins que, à la suite notamment d'une série d'expérimentations qui semblent confirmer en grande partie les intuitions théoriques de Bohr (v. à ce sujet Einstein, Podolsky et Rosen 1935, Bohr 1935, Bitbol 1996 : 249-254 / Aspect et al. 1982a, 1982b, Zeilinger 1997, Walther et al. 2005, Zeilinger et al. 2008a, 2008b etc.), un consensus assez large commence désormais à se dégager sur le fait que l'avènement de la mécanique quantique a rendu définitivement caduque la vision «multitudiniste » et atomistique de la nature (v. d'Espagnat $2002: 23$, Bitbol $1998: 212-230$ ) que nous avait léguée la physique classique : plus personne (ou presque) ne croit encore à l'heure actuelle dans la communauté des physiciens que les particules (sub)atomiques seraient des corpuscules distinctement individués aux contours « clairs et distincts ", susceptibles (comme les grains de sable ou les boules de billard) d'être localisés à tout moment dans un continuum spatiotemporel quadri-dimensionnel. Ce consensus va bien au-delà des tenants de l'interprétation de Copenhague, comme en témoignent par exemple les remarques de Roger Penrose (1992 : 325), d'Ilya Prigogine (1996 : 158), de Jean Bricmont [d'Espagnat (dir. 2002 : 33, 66] etc. On notera du reste que la principale alternative à l'interprétation « orthodoxe » qui existe à ce jour, à savoir la théorie de l'onde pilote mise au point par David Bohm (v. notamment Bohm et Hiley 1993), ne donne pas lieu à une description intégralement «multitudiniste » des phénomènes microphysiques [v. p. ex. d'Espagnat 2002 : 97-98, 142-144, d'Espagnat (dir.) 2002 : 41-43]: ses adeptes, Bohm en tête, ont fini par admettre en substance que le "réel » microphysique n'est pas atomistique (cf. p. ex. Bohm 1987: 39) mais se présente comme une totalité insécable (Bohm 1987 : 155 et passim), dans la mesure où il obéit à « un ordre implicite holistique et non spatio-temporel " (Bitbol 1998 : 234). Ce qui semble donc fondamentalement remis en cause par l'avènement de la mécanique quantique, c'est le principe de la «divisibilité par la pensée» (d'Espagnat 2002: 72; v. aussi d'Espagnat 1994: 123-127) de l'univers microphysique, ce dernier étant caractérisé (fût-ce de manière négative) par un trait distinctif fondamental: sa dimension «non locale», qui se traduit notamment par des interactions instantanées entre des particules suffisamment éloignées dans l'espace pour qu'une hypothétique relation d'influence causale qui se serait propagée de l'une à l'autre à une vitesse n'excédant pas celle de la lumière ne puisse être observée avant l'écoulement d'un laps de temps expérimentalement mesurable (v. n. 4 infra, d'Espagnat $2002: 378-380$ ).

2. V. d'Espagnat $1985: 31$ et passim, $2002: 111,114,120$.

3. D'où la remarque d'Erwin Schrödinger qu'«il vaut mieux ne pas regarder une particule comme une entité permanente mais plutôt comme un événement instantané » (Schrödinger 1992 : 47). Des physiciens comme Bernard d'Espagnat vont même désormais plus loin que Schrödinger, en postulant que le formalisme quantique ne saurait être interprété "comme fournissant la probabilité que tel ou tel événement arrive, et ne peut l'être que comme exprimant celle de tel ou tel résultat d'observation " (d'Espagnat 2002:170; v.d'Espagnat 2002: 116 et passim). Ce point de vue est toutefois contesté par d'autres chercheurs, qui considèrent que la mécanique quantique n'élimine pas - loin de là - la possibilité d'affirmer quelque chose à propos du « réel » microphysique "lui-même", en faisant valoir par exemple que des particules comme les électrons possèdent intrinsèquement une masse et une charge électrique.

4. Cf. infra, n. 13.

5. On se contentera de mentionner ici : (a) l'expérience bien connue des fentes d'Young (v. p. ex. Bitbol 1996 : 62-66), lors de laquelle un électron émis par une source située devant un cache opaque percé de deux fentes passe par les deux fentes en même temps ; (b) les expériences plus récentes d'Aspect et de Zeilinger (v. notamment Aspect et al. 1982a, 1982b, Zeilinger et al. 1997, Jennewein et al. 2002, Zeilinger et al. 2008a, 2008b ; cf. fig. 1), ces derniers ayant montré que deux photons "corrélés » se comportent en fait comme une seule et même entité, même s'ils sont distants (de plusieurs dizaines de kilomètres dans certaines expériences : cf. p. ex. Halder et al. 2005) dans l'espace. 
6. V. Bitbol $1998: 135-138$, d'Espagnat $2002: 366-368,449,451,457$.

7. On pourrait donc soutenir que le langage descriptif de la physique se présente aux yeux de Bohr comme une "voix » au sens bakhtinien du terme, autrement dit un ensemble de données discursives véhiculant un point de vue spécifique sur le monde qui en constitue en quelque sorte l'arrière-plan constitutif (v. Bakhtine 1978 : 113 et passim).

8. Mot-valise découlant de la fusion des lexèmes « onde » et « ubiquité ».

9. Du grec $\pi \alpha v \tau$ to í́ $\alpha$, littéralement « présence dans n’importe quel lieu ».

10. Penrose souligne ainsi que le formalisme mathématique qui fonde les théories physiques n'est pas pour lui une simple construction de l'esprit humain, mais renvoie à des vérités intemporelles « dont l'existence est tout à fait indépendante des activités des mathématiciens » (Penrose 1992 : 103).

11. Les spécialistes de la théorie quantique des champs ou encore des théories de la « décohérence » auront ainsi tendance à être plus critiques à l'égard du « multitudinisme » que ceux de la physique des particules (v. n. 12) : en témoigne par exemple un article célèbre de HansDieter Zeh (1993), éloquemment intitulé «There are no quantum jumps, nor are there particles »!

12. Qui ont conduit certains spécialistes de la théorie quantique des champs à évacuer toute référence à notre vision intuitive du « réel » dans la description des particules microphysiques, en définissant ces dernières de manière purement abstraite par le recours à des concepts mathématiques comme le «vecteur d'état de l'espace de Fock» (cf. Bitbol 1996 : 392-399, 1998 : 217-218).

13. Bernard Walliser (1977: 117) définit les concepts d'isomorphisme et d'homomorphisme comme suit: "On dira que deux systèmes sont homomorphes s'il existe une correspondance surjective entre caractéristiques de l'un et caractéristiques de l'autre. On dira que deux systèmes sont isomorphes lorsque cette correspondance est bijective ».

\section{RÉSUMÉS}

Cette étude porte sur l'importance accordée aux images verbales dans le discours descriptif utilisé en mécanique quantique, et plus précisément sur la conception de la langue scientifique qui est celle de Niels Bohr (1885-1962) : en raison d'une série de considérations techniques, méthodologiques et épistémologiques que nous nous proposons d'analyser in extenso, Bohr considère effectivement que les phénomènes subatomiques ne peuvent être évoqués directement (sans référence au contexte observationnel), par le biais d'un langage dénotatif non figural, mais uniquement de manière métaphorique, détournée, ce qui réduit à ses yeux le discours descriptif des physiciens à "des images et des paraboles». En examinant les textes de Bohr à la lumière d'un certain nombre de travaux épistémologiques, de commentaires et d'expérimentations auxquels ils ont donné lieu ultérieurement, nous nous proposons de décrire les implications conceptuelles d'une telle prise de position, qui constitue une vraie révolution sur le plan philosophique. 
INDEX

Index chronologique : XXe siècle

Mots-clés : physique quantique, holisme, complémentarité, non localité, langage figural

Index géographique : Europe

\section{AUTEUR}

\section{ILIAS YOCARIS}

Maitre de conférences en littérature française, Université de Nice-Sophia Antipolis, CIRCPLES EA3159

y.ilias@wanadoo.fr 